\title{
Reversible pulmonary trunk banding: VII. Stress echocardiographic assessment of rapid ventricular hypertrophy in young goats
}

\author{
Gustavo A. G. Fávaro, MD, PhD, ${ }^{a}$ Renato S. Assad, MD, PhD, ${ }^{\mathrm{b}}$ Maria C. D. Abduch, VMD, PhD, \\ Gustavo J. J. Silva, PE, PhD, ${ }^{\mathrm{b}}$ Guilherme S. Gomes, MD, ${ }^{\mathrm{b}}$ José L. Andrade, MD, PhD, ${ }^{\mathrm{a}}$ \\ José E. Krieger, $\mathrm{MD}, \mathrm{PhD},{ }^{\mathrm{b}}$ and Luiz Felipe P. Moreira, $\mathrm{MD}, \mathrm{PhD}^{\mathrm{b}}$
}

\begin{abstract}
Background: Ventricle retraining with abrupt systolic overload can cause myocardial edema and necrosis, followed by late ventricular failure. Intermittent systolic overload could minimize the inadequacy of conventional pulmonary artery banding. The present study compared ventricle function under dobutamine stress in 2 protocols of systolic overload in young goats.
\end{abstract}

\begin{abstract}
Methods: Nineteen young goats were divided into 3 groups: sham $(n=7$; no systolic pressure overload), continuous ( $\mathrm{n}=6$; systolic overload maintained for 96 hours), and intermittent ( $\mathrm{n}=6 ; 4$ periods of 12 -hour systolic overload, paired with a 12-hour resting period). Echocardiographic and hemodynamic evaluations were performed daily. The myocardial performance index and ejection fraction were evaluated at rest and during dobutamine stress. The goats were then killed for morphologic evaluation.
\end{abstract}

Results: The intermittent group underwent less systolic overload than the continuous group $(P<.05)$. Nevertheless, both groups had increased right ventricular and septal masses compared with the sham group $(P<.0002)$. Echocardiography revealed a major increase in right ventricular wall thickness in the intermittent group $(+64.8 \% \pm 23.37 \%)$ compared with the continuous group $(+43.9 \% \pm 19.26 \% ; P=.015)$. Only the continuous group remained with significant right ventricular dilation throughout the protocol $(P<.001)$. The intermittent group had a significantly better myocardial performance index at the end of the protocol, under resting and dobutamine infusion, compared with the continuous group $(P<.012)$.

Conclusions: Both systolic overload protocols have induced rapid right ventricular hypertrophy. However, only the intermittent group had better preservation of right ventricular function at the end of the protocol, both at rest and during dobutamine infusion. (J Thorac Cardiovasc Surg 2013;145:1345-51)

\section{Supplemental material is available online.}

In developing countries, the number of patients with transposition of the great arteries (TGA) presenting beyond the neonatal period is still considerable. In this scenario, complete anatomic repair must be preceded by left ventricle retraining with pulmonary artery banding, to enable the ventricle to handle the systemic circulation. Concern is increasing about the quality of subpulmonary ventricle retraining, aiming at the most physiologic hypertrophic process to avoid late ventricular dysfunction. ${ }^{1}$

\footnotetext{
From the Radiology Institute ${ }^{\mathrm{a}}$ and Heart Institute, ${ }^{\mathrm{b}}$ University of São Paulo Medical School, São Paulo, Brazil.

The present study was supported by the São Paulo State Foundation for Research Support; banding devices were provided by SILIMED Inc (Rio de Janeiro, Brazil). Disclosures: Authors have nothing to disclose with regard to commercial support. Received for publication Feb 18, 2012; revisions received April 20, 2012; accepted for publication July 26, 2012; available ahead of print Aug 27, 2012.

Address for reprints: Gustavo A. G. Fávaro, MD, PhD, Radiology Institute, Division of Echocardiography, University of São Paulo Medical School, Avenue Dr Enéas de Carvalho Aguiar, 255, $3^{\circ}$ Andar, Cerqueira César, São Paulo, SP 05403-001 Brazil (E-mail: guzfavaro@hotmail.com).

$0022-5223 / \$ 36.00$

Copyright (c) 2013 by The American Association for Thoracic Surgery http://dx.doi.org/10.1016/j.jtcvs.2012.07.068
}

Previous studies have looked for better alternatives to subpulmonary ventricle retraining. It has been demonstrated in a young animal model that intermittent systolic overload, in agreement with athletic philosophy, has promoted more prominent hypertrophy, compared with continuous systolic overload. ${ }^{2,3}$ However, these studies assessed global right ventricular (RV) systolic function only at rest, without showing any significant differences between the systolic overload protocols.

Nevertheless, the evaluation of RV function is difficult to image because of its complex morphology. Although cardiac magnetic resonance imaging is currently considered the reference technique for RV volumetry and calculation of the ejection fraction, various echocardiographic parameters can provide reliable information on RV dimensions and $\mathrm{RV}$ systolic and diastolic function in daily clinical practice. ${ }^{4}$ Therefore, the myocardial performance index (MPI) has been proposed as a relatively simple method to assess the combined systolic and diastolic performance of the right ventricle simultaneously, at rest and under dobutamine stress. $^{5}$

The present study aimed at a more detailed echocardiographic assessment of subpulmonary ventricular function in 2 different protocols of systolic overload, using MPI and a pharmacologic stress technique. 


$$
\begin{aligned}
& \text { Abbreviations and Acronyms } \\
& \begin{aligned}
\text { LV } & =\text { left ventricular } \\
\text { MPI } & =\text { myocardial performance index } \\
\text { PAB } & =\text { pulmonary artery banding } \\
\text { PT } & =\text { pulmonary trunk } \\
\text { RV } & =\text { right ventricular } \\
\text { RVEDV } & =\text { RV end-diastolic volume } \\
\text { RVEF } & =\text { RV ejection fraction } \\
\text { RVMPI } & =\text { RV myocardial performance index } \\
\text { TGA } & =\text { transposition of the great arteries }
\end{aligned}
\end{aligned}
$$

\section{METHODS}

Nineteen young goats, aged 30 to 60 days were enrolled in the present study and divided into 3 groups of comparable weight $(P=.84)$ : sham $(\mathrm{n}=7$; weight, $12.00 \pm 2.65 \mathrm{~kg})$, continuous $(\mathrm{n}=6$; weight, $11.27 \pm$ $3.20 \mathrm{~kg})$, and intermittent $(\mathrm{n}=6$; weight, $11.98 \pm 1.07 \mathrm{~kg})$. All goats received humane care in accordance with the guidelines established by the Brazilian College of Animal Experimentation. The ethics committee for research protocols at the University of São Paulo School of Medicine reviewed and approved the present study (CAPPesq 0664/09).

\section{ANESTHESIA}

Anesthesia was induced with intramuscular ketamine (20 $\mathrm{mg} / \mathrm{kg})$ and maintained with ketamine $(1 \mathrm{mg} / \mathrm{kg}$ intravenously) and nembutal (5 mg/kg intravenously) on demand. The goats were intubated and mechanically ventilated (Harvard 708, South Natick, Mass). An electrocardiogram was recorded and blood pressure measurements were taken with computer software (ACQknowledge, version 3.01; Biopac Systems, Inc, Goleta, Calif). Antibiotic therapy (cephazolin $500 \mathrm{mg}$ intravenously and gentamicin $40 \mathrm{mg}$ intramuscularly) was administered just before the operation and maintained during the protocol. Digoxin $(0.01 \mathrm{mg} / \mathrm{kg})$ and heparin (5000 IU) were also administered throughout the protocol. All the goats were extubated right after the surgical procedure and remained ambulatory and breathing spontaneously throughout the protocol.

\section{SURGICAL PROCEDURE}

The chest was opened at the fourth left intercostal space to expose the RV outflow tract, after lung retraction. A 17gauge heparinized catheter was inserted in the RV outflow tract, pulmonary trunk (PT), and descending aorta for pressure measurements at specific intervals during the entire study. The adjustable pulmonary artery banding (PAB) system (SILIMED; Silicone e Instrumental Medico-Cirurgico e Hospitalar Ltda, Rio de Janeiro, Brazil) was implanted just beyond the pulmonary valve, as previously described. ${ }^{6}$

\section{RV SYSTOLIC OVERLOAD PROTOCOL}

RV training was begun after a 72-hour convalescence period with percutaneous PAB insufflation with saline solution to achieve an $\mathrm{RV} /$ systemic pressure ratio of 0.7 , limited by a $10 \%$ decrease in systolic blood pressure. Readjustments were made every morning throughout the protocol. If systemic hypotension and/or respiratory distress developed after PAB inflation, it was deflated to a volume compatible with RV tolerance and maintenance of goat hemodynamics. A 96-hour study period has been previously established as the minimum time required for cardiac masses equalization in young goats. ${ }^{7}$

\section{Continuous Group Protocol}

In the continuous group, the goats remained with continuous systolic overload for 96 hours, with daily assessment to keep the RV/aortic pressure ratio at 0.7 . Hemodynamic data were collected once daily (mornings) during PAB readjustments.

\section{Intermittent Group Protocol}

In the intermittent group, the goats underwent 4 daytime periods of 12-hour systolic overload, alternating with a 12-hour nighttime resting period. Hemodynamic data were collected every 12 hours, during PAB readjustments.

\section{Sham Group Protocol}

In the sham group, the PAB system was maintained deflated during the entire protocol. Hemodynamic data were collected once daily (mornings).

\section{ECHOCARDIOGRAPHY}

All the goats while under light sedation (ketamine $15 \mathrm{mg}$ intramuscularly) were examined by a single experienced observer preoperatively and daily throughout the study and monitored continuously with surface electrocardiography. The following echocardiographic parameters were studied using multifrequency transducers (7.5 and 2.5 $\mathrm{MHz}$, Acuson Cypress; Siemens, Erlagen, Germany): left ventricular (LV), RV, and ventricular septum wall thicknesses, RV end-diastolic volume (RVEDV) and RV endsystolic volume, RV end-diastolic diameter, RV ejection fraction (RVEF), and RV MPI (RVMPI).

Because of the keel structure of the goat thorax, the RV free wall thickness was taken from the parasternal longaxis 4-chamber view and from the parasternal short-axis view (at basal and papillary muscle levels), where the limits of the RV free wall were more easily obtained. The LV enddiastolic posterior and septal wall thicknesses were measured through the parasternal long-axis view, at the level of the mitral valve leaflet tips. All the wall thicknesses were measured using 2-dimensional technique, under resting conditions.

Also, from the long-axis, 4-chamber view, the RVEF was measured by the modified Simpson rule. The RVMPI was calculated from pulsed wave Doppler of RV inflow and outflow tracts, positioning the sample volume at the level of the tricuspid and pulmonary valve leaflet tips, respectively, as previously described by Ishii and colleagues. ${ }^{8}$ This index 
expresses the ratio between isovolumic times (contraction and relaxation) and represents the assessment of RV systolic and diastolic function. It was obtained at rest and during pharmacologic stress with dobutamine, according to the following formula: (IVCT + IVRT) $\times \mathrm{ET}^{-1}$, where IVCT represents the isovolumetric contraction time; IVRT, the isovolumetric relaxation time; and ET, the ejection time. The values were obtained through the long-axis, 4-chamber view, using the Doppler method, by positioning the volume sample at the center of the tricuspid valve and measuring the interval between the onset of valve closure and the beginning of the next diastole (time a).

The RV ejection time was calculated with the volume sample located in the RV outflow tract (time b) through the parasternal short-axis view. The value of isovolumetric contraction time + isovolumetric relaxation time was obtained by subtracting time a from time $b$. This result was then divided by the ejection time, providing the RVMPI value. The mean of 3 consecutive measurements were then obtained to achieve the final RVMPI value for each goat.

The assessment of RV function under dobutamine infusion was performed during 2 periods: before the surgical procedure (baseline) and at protocol end (96 hours). During the stress echocardiographic examination, a graded dobutamine infusion was started at $5 \mu \mathrm{g} / \mathrm{kg} / \mathrm{min}$ and increased at 3-minute intervals to $10,20,30$, and $40 \mu \mathrm{g} / \mathrm{kg} / \mathrm{min}^{9}{ }^{9}$ The endpoints were the achievement of a target heart rate, defined as 1.7 of the baseline heart rate. When the target heart rate was achieved, the echocardiographic parameters RVEF and RVMPI were measured.

\section{MORPHOLOGIC ASSESSMENT}

The goats were humanely killed after 96 hours of the study protocol. After harvesting the heart, the pericardial fat, both atria, and semilunar valves were dissected from it. The right ventricle, left ventricle, and ventricular septum were separated using the Fulton technique, individually weighed (METTLER AE-200; Mettler-Toledo AG, Greifensee, Switzerland), and indexed to each goat's body weight. The water content was obtained individually in each cardiac chamber by subtracting the collected sample weight at necropsy from the weight of the dehydrated chamber $\left(70\right.$ hours at $\left.60^{\circ} \mathrm{C}\right)$. Values were obtained as a percentage of weight change.

\section{STATISTICAL ANALYSIS}

The data are presented as the mean \pm standard deviation. A comparison of variables was performed with 2-way analysis of variance, except for body weight, wall masses, and water content, which were performed with 1-way analysis of variance. Both analyses were followed by the Bonferroni post hoc test. The systolic overload imposed on the right ventricle of the continuous and intermittent groups was assessed by calculating the areas under the curve (trapezoidal method). The comparison between these areas was performed using the unpaired Student $t$-test. Statistical analysis was performed using GraphPad Prism, version 4 (GraphPad Prism, La Jolla, Calif) and SigmaStat, version 3.11.0 (Systat Software, Inc, Chicago, Ill).

\section{RESULTS}

\section{Hemodynamic Measurements}

The systolic RV-PT gradient increased in both study groups $(P<.001)$ and in every measurement period $(P<.001)$. It was greater than that in the sham group, from the beginning of RV systolic overload (0 hour) until the end of the protocol $(P<.001)$. The area under the RV-PT gradient curve was calculated, as a method of measuring the RV systolic overload imposed on each group. The systolic overload of the continuous group (4597 $\pm 814 \mathrm{~mm}$ $\mathrm{Hg} \times \mathrm{h})$ and intermittent group $(2777 \pm 384.6 \mathrm{~mm} \mathrm{Hg} \times \mathrm{h})$ was greater than that in the sham group $(480 \pm 101.10 \mathrm{~mm}$ $\mathrm{Hg} \times \mathrm{h}$ ). The continuous group was the most exposed to systolic overload $(P<.05)$. Parallel to the RV-PT gradient, both study groups showed an increased RV/aorta systolic pressure ratio over time (Table E1) compared with its respective baseline value and with that in the sham group at the same point $(P<.001)$.

\section{Echocardiographic Findings at Rest}

Both continuous and intermittent groups had an increased $\mathrm{RV}$ free wall thickness compared with the preoperative measurements and the sham group at 72 and 96 hours $(P<.001)$. However, the RV thickness increase was significantly greater in the intermittent group at the end of the protocol $(5.38 \pm 0.82 \mathrm{~mm})$ compared with that in the continuous $(4.65 \pm 0.48 \mathrm{~mm})$ and sham $(3.36 \pm 0.08 \mathrm{~mm})$ groups $(P=.001)$. No differences were seen in the septum and LV thicknesses among the 3 groups $(P=.10)$ or throughout the protocol $(P>.82)$. The graph showing the $\mathrm{RV}$ free wall thickness changes throughout the protocol is available on-line (Figure E1).

$\mathrm{RV}$ end-diastolic volume changes were significantly different between the 2 groups throughout the protocol $(P<.001)$. Both study groups showed a significant increase in RVEDV immediately after the onset of RV systolic overload (intermittent, $112.33 \% \pm 87.24 \%, P=.002$; and continuous, $98.3 \% \pm 123.5 \%, P=.007)$. At 24 hours, the intermittent group had recovered RVEDV to a value comparable to that in the sham group, but in the continuous group, the RVEDV continued to be significantly increased at 24 $(101.1 \% \pm 70.3 \%, P=.03), 72(87.6 \% \pm 87.3 \%$, $P=.03)$, and $96(92.7 \% \pm 89.9 \%, P=.015)$ hours compared with the sham group. The graph showing the RV enddiastolic volume changes is available on-line (Figure E2).

The RVEF changes throughout the study period were significantly different between groups $(P<.001)$ and over time $(P<.001)$. Both study groups had significant RVEF 

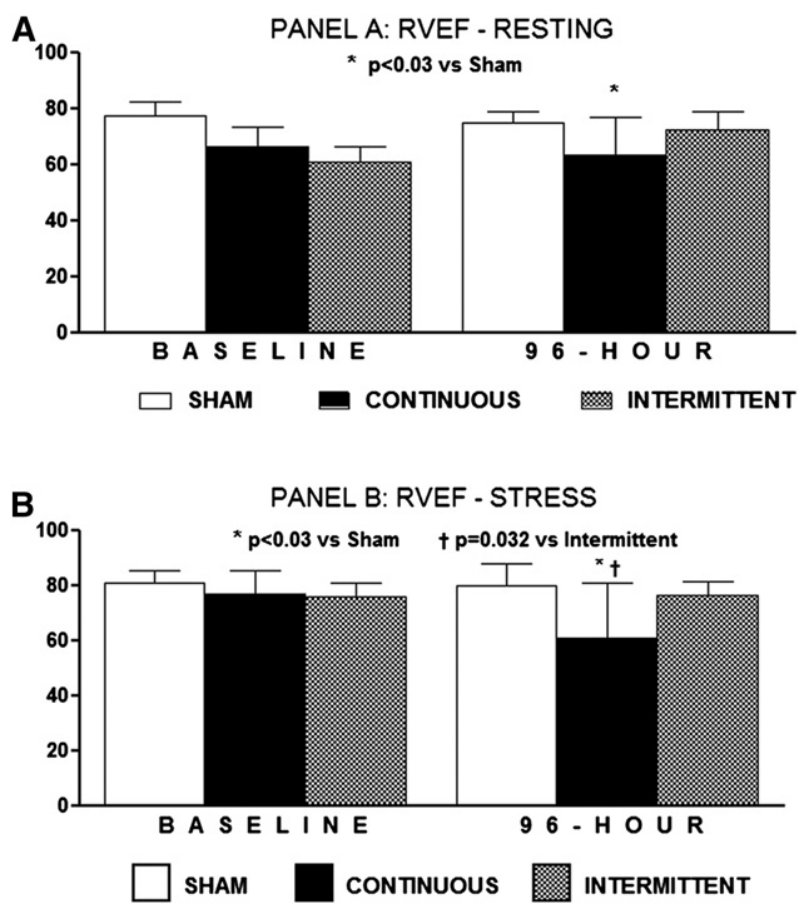

FIGURE 1. Right ventricular ejection fraction $(R V E F)$ of sham, continuous, and intermittent groups, under dobutamine stress. A, Resting condition $(* P<.03$ compared with that in sham group at measurement point) and, B, dobutamine stress $(* P<.03$ compared with that in sham group at measurement point). $\dagger P=.032$ compared with that in intermittent group at measurement point.

impairment immediately after the onset of RV systolic overload (intermittent, $-47.7 \% \pm 26.9 \%, P=.001$; and continuous, $-39.6 \% \pm 27.8 \%, P=.002$ ). Subsequently, the intermittent-group RVEF recovered to values comparable to those in the sham group, and the continuous group maintained prolonged RVEF impairment at $24(-38.8 \% \pm$ $23.1 \%, P<.001)$ and $48(-19.6 \% \pm 32.4 \%, P=.029)$ hours compared with the sham group. After 72 hours of systolic overload, the RVEF in both study groups had recovered to values comparable to those in the sham group. The RVEF data of the 3 groups throughout the study period are shown on line in Table E2 and Figure E3.

In contrast to the RVEF findings, the RVMPI values in the continuous group were worse after 72 hours $(0.38 \pm 0.17)$ and 96 hours $(0.41 \pm 0.28)$ of systolic overload, with a significant difference from that in the sham group at 72 hours $(0.16 \pm 0.08 ; P=.009)$ and from both the sham $(0.22 \pm$ $0.07 ; P=.039)$ and intermittent $(0.10 \pm 0.03 ; P<.001)$ groups at 96 hours. These data are also available on-line (Table E3 and Figure E4).

\section{Echocardiographic Findings Under Pharmacologic Stress}

Dobutamine infusion caused a similar heart rate increase in the 3 groups, at both baseline $(P=.66)$ and the end of the
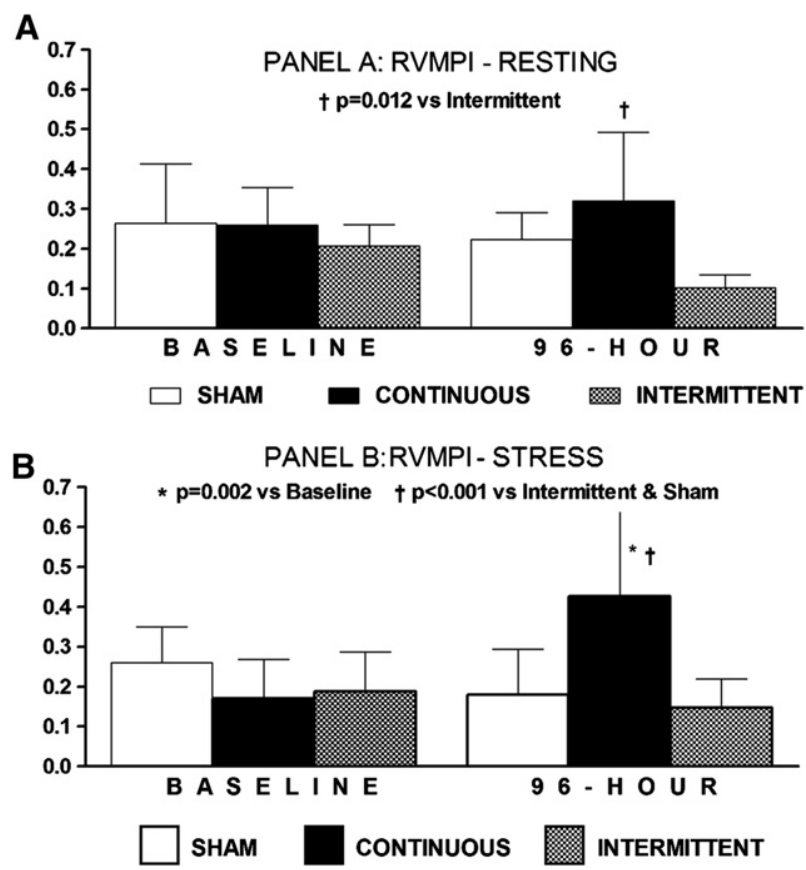

FIGURE 2. Right ventricular myocardial performance index (RVMPI) of sham, continuous, and intermittent groups, under dobutamine stress. A, Resting condition $(\dagger P=.012$ compared with that in intermittent group at measurement point) and, B, dobutamine stress $(* P=.002$ compared with baseline value; $\nmid P<.001$ compared with that in intermittent and sham groups at measurement point).

protocol $(P=.10)$. The heart rate data are available on-line (Table E4).

Figure 1 shows the RVEF before surgical intervention (baseline) and at the end of the protocol, at rest (Figure 1, $A$ ) and under pharmacologic stress (Figure 1,B). Although the 3 groups completed the protocol with a normal RVEF at rest, the continuous group had the smallest RVEF value $(63.00 \% \pm 13.90 \%)$ compared with that in the sham group $(74.71 \% \pm 4.19 \% ; P=.026)$. It was observed that the RVEF in the intermittent group had improved after the ventricular training period $(72.33 \% \pm 6.28 \%)$, relative to its baseline condition $(60.83 \% \pm 5.34 \% ; P=.013)$. During the dobutamine stress test, the RVEF in the 3 groups remained within the normal range. However, the continuous group again had the lowest RVEF $(60.67 \% \pm 20.30 \%)$ compared with that in the sham $(79.57 \% \pm 8.02 \%$; $P=.026)$ and intermittent $(76.33 \% \pm 4.97 \% ; P=.032)$ groups.

The RVMPI graph of the 3 groups during baseline and at 96 hours, at rest and under dobutamine stress is shown in Figure 2. The baseline values were similar among the 3 groups, with or without dobutamine. However, the continuous group had unfavorable RVMPI rates at the end of the protocol, both at rest $(0.32 \pm 0.17)$ and with dobutamine stress $(0.43 \pm 0.21)$ compared with the intermittent group during rest $(0.10 \pm 0.03 ; P<.012)$ and under 
TABLE 1. Cardiac mass weight of sham, continuous, and intermittent groups, indexed to goat body weight

\begin{tabular}{lccc}
$\begin{array}{c}\text { Cardiac mass } \\
(\mathbf{g} / \mathbf{k g})\end{array}$ & $\begin{array}{c}\text { Sham } \\
(\mathbf{n}=\mathbf{7})\end{array}$ & $\begin{array}{c}\text { Continuous } \\
(\mathbf{n}=\mathbf{6})\end{array}$ & $\begin{array}{c}\text { Intermittent } \\
(\mathbf{n}=\mathbf{6})\end{array}$ \\
\hline Right ventricle & $0.76 \pm 0.12$ & $1.21 \pm 0.11^{*}$ & $1.15 \pm 0.07^{*}$ \\
Septum & $0.86 \pm 0.10$ & $1.18 \pm 0.12^{*}$ & $1.04 \pm 0.10^{*}$ \\
Left ventricle & $1.38 \pm 0.17$ & $1.45 \pm 0.19$ & $1.53 \pm 0.27$ \\
\hline
\end{tabular}

Data presented as mean \pm standard deviation. $* P<.05$ compared with sham group.

pharmacologic stress $(0.15 \pm 0.07 ; P<.012)$. Compared with the sham group during dobutamine infusion (RVMPI, $0.18 \pm 0.11)$, the RVMPI of the continuous group increased significantly $(P<.001)$.

\section{Morphologic Findings}

Cardiac mass weight. Both continuous and intermittent systolic overload resulted in significant increases in RV (intermittent, $51.3 \%$; continuous, $59.2 \% ; P<.05)$ and septal masses (intermittent, $20.9 \%$; continuous, $37.2 \% ; P=.05$ ) compared with those in the sham group (Table 1). No significant changes were noted in LV mass $(P=.4662)$.

Myocardial water content. A minor, although significant, increase was seen in the water content of the RV myocardium of the continuous $(+3.2 \%)$ and intermittent $(+3.0 \%)$ groups $(P=.0102)$ compared with that in the sham group. Similarly, increased water content was observed in the septum of the continuous $(+2.9 \%)$ and intermittent $(+2.4 \%)$ groups $(P=.0028)$ compared with that in the sham group. These data are available on-line in Table E5.

\section{DISCUSSION}

In the present study, we aimed to assess subpulmonary ventricular function using 2 pulmonary trunk banding protocols, focusing on a more sensitive method to detect early disturbances in ventricular function during the process of rapid RV hypertrophy. In addition to Simpson's method, we used the MPI to evaluate ventricular function with a greater sensitivity and accuracy, especially in goats, which usually have poor imaging windows because of their keelshaped chest. The combination of RVMPI calculation, which did not require morphologic analysis of the heart chamber or the perfect visualization of endocardial borders, with stress echocardiography resulted in significant improvement in ventricular function assessment in this rapid ventricular hypertrophy protocol. It was clearly demonstrated that intermittent systolic overload, although quantitatively smaller than continuous overload, has promoted functionally superior hypertrophy under dobutamine stress at the 96-hour study period. Even at rest, the intermittent group had a significantly more favorable RVMPI than the continuous group had after the training period. However, both study groups evolved with important RVEF impairment immediately after starting the protocol, owing to acute systolic overload of $70 \%$ systemic pressure, which resulted in RV systolic dysfunction earlier in the protocol. In contrast to the RVMPI results, the initial RVEF worsening recovered by the end of the protocol in both study groups, to values comparable to those in the sham group, with or without dobutamine infusion. Therefore, systolic dysfunction might have occurred in both study groups at the beginning, with diastolic dysfunction only in the continuous group at the end of the protocol. RVMPI worsening might have been caused by a diastolic disorder, although it is not possible to quantify isolated systolic or diastolic index changes. At the end of protocol, the difference between the 2 echocardiographic methods was more evident with the MPI, which should indicate a greater sensitivity for assessing ventricular function and enhance diagnostic confidence in ventricular retraining.

Regarding the RV end diastolic volume, continuous systolic overload resulted in persistent dilation throughout the protocol. This could also reflect worse ventricular adaptation in the continuous group. However, the 12-hour resting periods might have optimized subendocardial coronary flow in the intermittent group and, consequently, replenished energy substrates to the myocardial hypertrophy process, limiting the severity of systolic overload. That would probably provide a better adaptation and consequent preservation of ventricular function in the intermittent group at periods of systolic overload.

MPI has been used in a wide variety of heart diseases in adults and children, including congenital univentricular heart lesions and in the follow-up of cavopulmonary connections. ${ }^{10}$ Its use has recently been reported in fetal echocardiography. ${ }^{11}$ Previous studies from Tei and colleagues ${ }^{12}$ have demonstrated the usefulness of the MPI in the evaluation of systolic and diastolic dysfunction in patients with idiopathic dilated cardiomyopathy. In aortic stenosis, MPI is used to assess both the severity of aortic stenosis and the presence of LV contractile reserve. ${ }^{13}$ It can provide important prognostic information in patients with associated LV systolic dysfunction, because surgical treatment with aortic valve replacement appears to improve the outcome for most patients with LV contractile reserve. In contrast, surgery is associated with high mortality in the absence of a contractile reserve. Similarly, we can derive the same MPI capacity to differentiate goats with RV systolic overload, artificially induced by PAB, among those with or without preserved ventricular function.

The results of the present study might reflect the morphologic findings of Carroll and colleagues ${ }^{14}$ in pigs that underwent continuous systolic overload for 7 hours. They found intense inflammatory infiltrate in the myocardium, with foci of cellular necrosis in varying degrees and subsequent late ventricular dysfunction, probably related to unbalanced oxygen demand in the hypertrophic myocardium. Evidence has shown that abrupt systolic overload can evolve, with 
systolic dysfunction associated with diastolic disorder or just isolated filling restriction, as demonstrated by Leeuwenburgh and colleagues. ${ }^{15}$ They have demonstrated decreased cardiac output in young sheep that underwent $\mathrm{PAB}$, despite the better RV contractility. This divergence is probably related to diastolic dysfunction, by increased $\mathrm{RV}$ relaxation time and decreased ventricular compliance. Therefore, the assessment of diastolic function should ideally be a part of the echocardiographic examination during subpulmonary ventricle retraining.

\section{Morphologic Versus Echocardiographic Assessment of Hypertrophy}

According to the echocardiographic analysis, the intermittent group had a greater RV free wall thickness than the continuous group, despite less exposure to systolic overload. These data were not supported by the morphologic analysis. Both protocols promoted an increase in septal and RV masses of similar magnitude in the study groups. Perhaps, the greater RV dilation observed by echocardiography in the goats in the continuous group influenced the interpretation of the RV free wall thickness. Regarding myocardial water content, the mild increase observed in the septum and right ventricle in both trained groups would not justify the increased myocardial mass weight, suggesting that it was probably related to increased protein synthesis and cell proliferation triggered by systolic overload and not to cellular and/or interstitial edema, as previously documented by Abduch and colleagues. ${ }^{16}$

\section{Clinical Implications}

The preparation of the subpulmonary ventricle, required to perform the 2-stage Jatene operation beyond the neonatal period, is still considered an alternative approach in several pediatric cardiology centers worldwide. According to Kang and colleagues, ${ }^{17}$ late diagnosis of congenital heart disease is remarkable in several countries and up to $95 \%$ of infants remain untreated. However, the echocardiographic, hemodynamic, and clinical criteria in decision making for the optimal surgical treatment of TGA beyond the neonatal period are still controversial. The presence of LV collapse on echocardiography, a ventricular mass index less than $35 \mathrm{~g} / \mathrm{m}^{2}$, $\mathrm{LV} /$ systemic blood pressure ratio less than 0.67 , and age older than 3 weeks are parameters suggested by several investigators to select candidates for ventricular retraining before anatomic repair. ${ }^{18,19}$ In addition to the controversy about those parameters and the quality of myocardial tissue hypertrophy, the indication for the 2-stage operation has been debated. Several centers have reported satisfactory results with primary anatomic repair in unfavorable cases using mechanical circulatory support devices or extracorporeal membrane oxygenation. ${ }^{20}$ Nevertheless, the obtained results have still been difficult to reproduce in other centers. Notwithstanding the uncertainties, with the current development of the ventricular retraining technique and because of the unavailability of the routine use of extracorporeal membrane oxygenation or ventricular assist devices in some cardiac centers, and the RV late dysfunction occurring after the Mustard and Senning operations, LV retraining remains as an important option for the 2-stage Jatene operation in selected cases. As mentioned previously, the high morbidity and mortality rates observed with traditional PAB are probably related to the intense and abrupt continuous pressure overload and can cause LV dysfunction, neoaortic regurgitation, and RV outflow tract obstruction. Therefore, intermittent systolic overload to prepare the subpulmonary ventricle, simulating the physical conditioning of athletes, might provide a better customization of ventricular retraining and improve its effects on myocardial function. Accordingly, a more physiologic hypertrophy might be achieved, aiming at preservation of future ventricular function. The results of the present study suggest that intermittent systolic overload can improve clinical management during LV retraining interstage for patients with TGA beyond the neonatal period and in those with congenitally corrected TGA or after atrial baffle operations with RV failure. It could favorably affect the results for a 2-stage Jatene operation.

\section{Study Limitations}

Considering the limitations of clinical inferences from observations of experimental studies, we cannot be certain sure that the behavior of the human left ventricle with TGA would act exactly the same as observed in the right ventricle of young goats. Although the architecture of the cardiomyocytes in the RV walls is comparable to that found in the left ventricle in terms of endocardial and epicardial helical angles, the right ventricle in both the normal and the hypertrophied state lacks the extensive zone of circular myocytes seen in the midportion of the LV walls. This architectural arrangement in a circular fashion can generate greater cavity pressures, with important implications in the systolic and diastolic functions of the left ventricle. ${ }^{21}$ Another important concern is that the continuous systolic overload imposed on the right ventricle was adjustable and not created with a traditional fixed band, commonly used in clinical practice. In the present study, the systolic overload might be less acute and intense using a percutaneously adjustable $\mathrm{PAB}$ than when using traditional $\mathrm{PAB}$, in which no changes are possible during ventricular retraining. Nevertheless, the coronary arterial circulation is not submitted to a hypertensive regimen, which is more closely related to the rapid 2-stage approach. ${ }^{16}$ Regarding the MPI, it is important to emphasize that no reference values have been established for goats. Even in children and neonates, a disparity exists between the normal values. ${ }^{22}$ In the present study, the baseline values were taken as a reference for subsequent analysis during the systolic overload protocol. 
Previous studies have shown that in the absence of an increased ventricular preload or myocardial infarction, there is a good correlation between the values measured by the MPI using conventional Doppler and tissue Doppler, which was not possible in these animals because of the absence of an apical 4-chamber view. ${ }^{23,24}$

\section{CONCLUSIONS}

The results of the present study have demonstrated that both systolic overload protocols promoted ventricular hypertrophy that was more evident in the intermittent group on echocardiography, despite less exposure to RV systolic overload. There was preservation of systolic and diastolic function in the intermittent group, both at rest and during dobutamine stress. In contrast, the functional performance of the continuous group was worse during rest and pharmacologic stress. The use of MPI in conjunction with stress echocardiography improved the accuracy of the diagnosis in assessment of dysfunction during subpulmonary ventricle retraining.

\section{References}

1. Boutin C, Wernovsky G, Sanders SP, Jonas RA, Castañeda A, Colan SD. Rapid two-stage arterial switch operation: evaluation of left ventricular systolic mechanics late after in acute pressure overload stimulus in infancy. Circulation. 1994;90:294-303.

2. Assad RS, Rodriguez MQ, Abduch MCD, Valente AS, Andrade JL, Krieger JE, Marcial MB. Adjustable pulmonary trunk banding: comparison of two methods of acute subpulmonary ventricle hypertrophy. Braz J Cardiovasc Surg. 2006;21: 418-28.

3. Assad RS, Atik FA, Oliveira FS, Fonseca-Alaniz MH, Abduch MC, Silva GJ, Favaro GG, Krieger JE, Stolf NA. Reversible pulmonary trunk banding. VI: glucose-6-phosphate dehydrogenase activity in rapid ventricular hypertrophy in young goats. J Thorac Cardiovasc Surg. 2011;142:1108-13.

4. Mertens LL, Friedberg MK. Imaging the right ventricle - current state of the art. Nature Rev Cardiol. 2010;7:551-63.

5. Norager B, Husic M, Muller JE, Bo Hansen A, Pellikka PA, Egstrup K. Changes in the Doppler myocardial performance index during dobutamine echocardiography: association with neurohormonal activation and prognosis after acute myocardial infarction. Heart. 2006;92:1071-6.

6. Valente AS, Assad RS, Abduch MC, Silva GJ, Thomaz PG, Miana LA, Krieger JE, Stolf NA. Pulmonary trunk reversible banding. IV: analysis of right ventricle acute hypertrophy in an intermittent overloading experimental model. Braz J Cardiovasc Surg. 2008;23:60-9.

7. Dias CA, Assad RS, Caneo LF, Abduch MC, Aiello VD, Dias AR, Marcial MB, Oliveira SA. Reversible pulmonary trunk banding. II. An experimental model for rapid pulmonary ventricular hypertrophy. J Thorac Cardiovasc Surg. 2002;124: 999-1006.

8. Ishii M, Eto G, Tei C, Tsutsumi T, Hashino K, Sugahara Y, et al. Quantitation of the global right ventricular function in children with normal heart and congenital heart disease: a right ventricular myocardial performance index. Pediatr Cardiol. 2000;21:416-21.

9. Mathias W Jr, Arruda A, Santos F, Arruda A, Mattos E, Osorio A, et al. Safety of dobutamine-atropine stress echocardiography: a prospective experience of 4,033 consecutive studies. J Am Soc Echocardiogr. 1999;12:785-91.

10. Zhang YQ, Sun K, Zhu SL, Wu LP, Chen GZ, Zhang ZF, et al. Doppler myocardial performance index in assessment of ventricular function in children with single ventricles. World J Pediatr. 2008;4:109-13.

11. Hamela-Olkowska A, Szymkiewicz-Dangel. Quantitative assessment of the right and left ventricular function using pulsed Doppler myocardial performance index in normal fetuses at 18 to 40 weeks of gestation. Ginekol Pol. 2011;82:108-13.

12. Tei C, Ling LH, Hodge DO, Bailey KR, Oh JK, Rodeheffer RJ, et al. New index of combined systolic and diastolic myocardial performance: a simple and reproducible measure of cardiac function — a study in normals and dilated cardiomyopathy. J Cardiol. 1995;26:357-66.

13. Bruch C, Dagres N, Katz M, Bartel T, Erbel R. Severe aortic valve stenosis with preserved and reduced systolic left ventricular function: diagnostic usefulness of the Tei index. J Am Soc Echocardiogr. 2002;15:869-76.

14. Carroll SM, Nimmo LE, Knoepfler PS, White FC, Bloor CM. Gene expression in a swine model of right ventricular hypertrophy: intracellular adhesion molecule, vascular endothelial growth factor and plasminogen activators are upregulated during pressure overload. J Mol Cell Cardiol. 1995;27:1427-41.

15. Leeuwenburgh BP, Steendijk P, Helbing WA, Baan J. Indexes of diastolic RV function: load dependence and changes after chronic RV pressure overload in lambs. Am J Physiol Heart Circ Physiol. 2002;282:1350-8.

16. Abduch MC, Assad RS, Rodriguez MQ, Valente AS, Andrade JL, Demarchi LM Aiello VD. Reversible pulmonary trunk banding III: assessment of myocardial adaptive mechanisms - contribution of cell proliferation. J Thorac Cardiovasc Surg. 2007;133:1510-6.

17. Kang N, de Leval MR, Elliot M, Tsang V, Kocyildirim E, Sehic I, et al. Extending the boundaries of the primary arterial switch operation in patients with transposition of the great arteries and intact ventricular septum. Circulation. 2004;110: 123-7.

18. Parker NM, Zuhdi M, Kouatli A, Baslaim G. Late presenters with dextrotransposition of great arteries and intact ventricular septum: to train or not to train the left ventricle for arterial switch operation. Congenit Heart Dis. 2009;4: 424-32.

19. Ismail SR, Kabbani MS, Najm HK, Abusuliman RM, Elbarbary M. Early outcome for primary arterial switch operation beyond the age of 3 weeks. Pediatr Cardiol. 2010;31:663-7.

20. Bisoi AK, Sharma P, Chauhan S, Reddy SM, Das S, Saxena A, Kothari SS. Primary arterial switch operation in children presenting late with d-transposition of great arteries and intact ventricular septum: when is it too late for a primary arterial switch operation? Eur J Cardiothorac Surg. 2010;38:707-13.

21. Nielsen E, Smerup M, Agger P, Frandsen J, Ringgard S, Pedersen M, et al. Normal right ventricular three-dimensional architecture, as assessed with diffusion tensor magnetic resonance imaging, is preserved during experimentally induced right ventricular hypertrophy. Anat Rec (Hoboken). 2009;292:640-51.

22. Cui W, Roberson DA. Left ventricular Tei index in children: comparison of tissue Doppler imaging, pulsed wave Doppler, and M-mode echocardiography normal values. J Am Soc Echocardiogr. 2006;19:1438-45.

23. Correale M, Totaro A, Ieva R, Brunetti ND, Di Biase M. Time intervals and myocardial performance index by tissue Doppler imaging. Intern Emerg Med. 2011; 6:393-402.

24. Tekten T, Onbasili AO, Ceyhan C, Unal S, Discigil B. Novel approach to measure myocardial performance index: pulsed-wave tissue Doppler echocardiography. Echocardiography. 2003;20:503-10. 


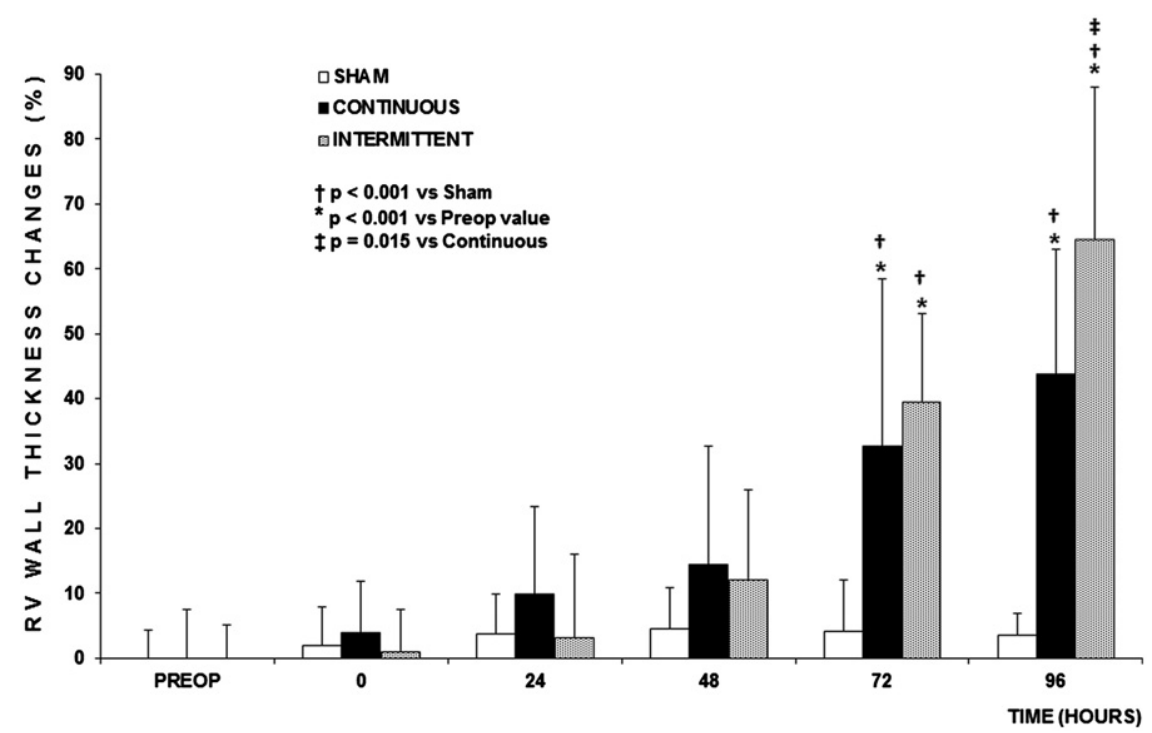

FIGURE E1. Right ventricular $(R V)$ thickness changes in sham, continuous, and intermittent groups throughout protocol. $\dagger P<.001$ compared with that of sham group; $* P<.001$ compared with respective baseline value; and $\ddagger P=.015$ compared with that in continuous group at same measurement point.

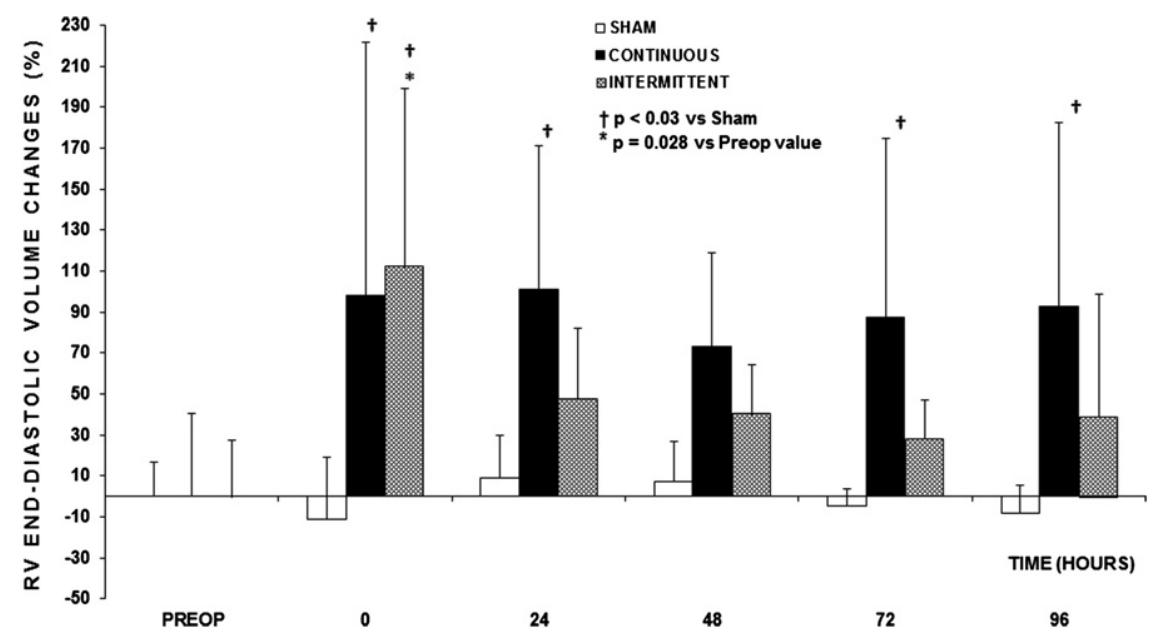

FIGURE E2. Right ventricular $(R V)$ end-diastolic volume changes in sham, continuous, and intermittent groups throughout protocol. $\dagger P<.03$ compared with that of sham group; ${ }^{*} P=.028$ compared with its respective preoperative (Preop) value. 


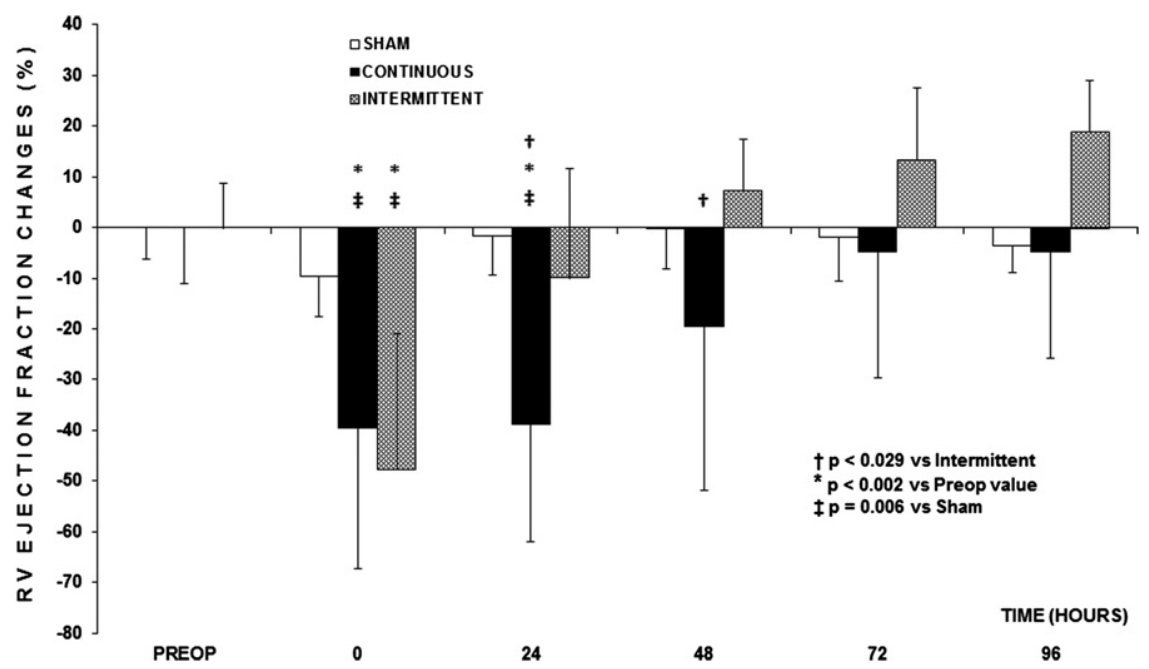

FIGURE E3. Right ventricular $(R V)$ ejection fraction changes $(\%)$ of sham, continuous, and intermittent groups throughout protocol, under resting conditions. $\dagger P<.029$ compared with that in intermittent group at same measurement point; $* P<.002$ compared with its respective preoperative $($ Preop $)$ value; and $\ddagger P=.006$ compared with that in sham group at same measurement point.

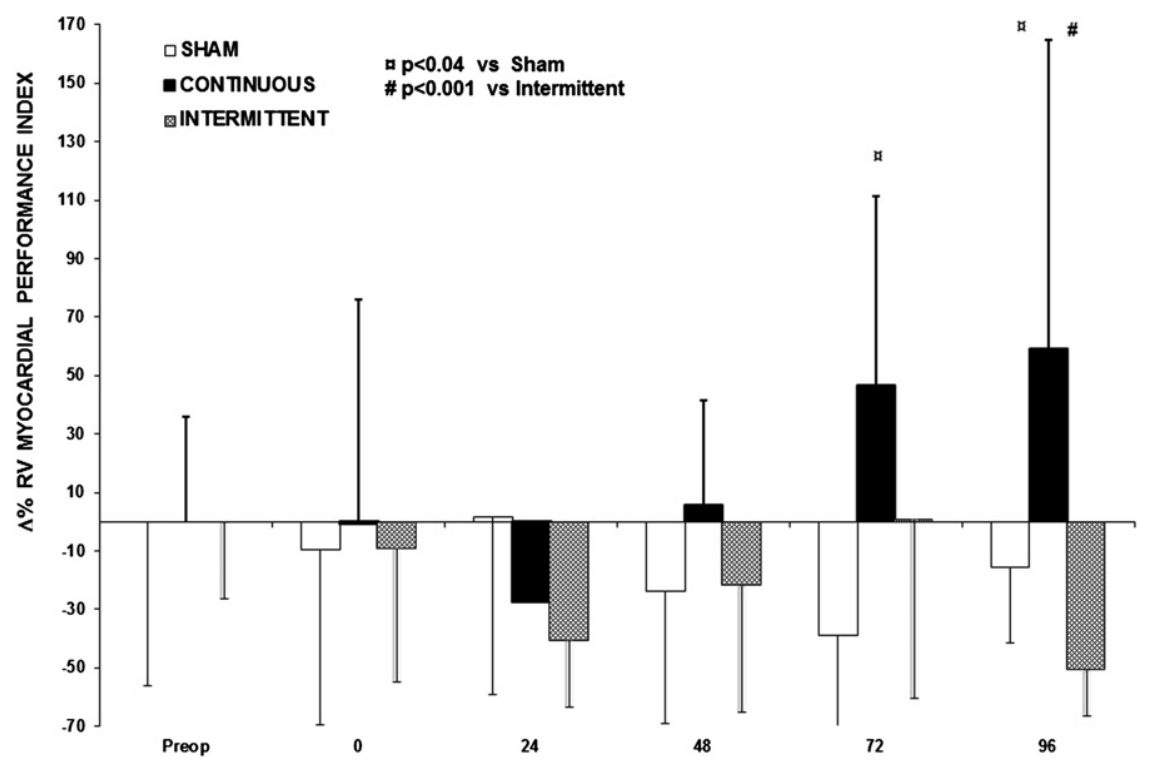

Time (hours)

FIGURE E4. Right ventricular $(R V)$ myocardial performance index changes (\%) of sham, continuous, and intermittent groups throughout protocol, under resting conditions. $\not P<.04$ compared with that in sham group at same measurement point; and $\# P<.001$ compared with that in intermittent group at same measurement point. 
TABLE E1. RV/aorta systolic pressure ratio of sham, continuous, and intermittent groups throughout protocol

\begin{tabular}{lccc}
\hline $\begin{array}{c}\text { Measurement } \\
\text { point }(\mathbf{h})\end{array}$ & $\begin{array}{c}\text { Sham } \\
(\mathbf{n}=\mathbf{7})\end{array}$ & $\begin{array}{c}\text { Continuous } \\
(\mathbf{n}=\mathbf{6})\end{array}$ & $\begin{array}{c}\text { Intermittent } \\
(\mathbf{n = 6})\end{array}$ \\
\hline Baseline & $0.36 \pm 0.06$ & $0.33 \pm 0.05$ & $0.32 \pm 0.05$ \\
0 & $0.36 \pm 0.06$ & $0.66 \pm 0.08^{*}$ & $0.70 \pm 0.06^{*}$ \\
24 & $0.34 \pm 0.06$ & $0.72 \pm 0.10^{*}$ & $0.75 \pm 0.05^{*}$ \\
48 & $0.33 \pm 0.08$ & $0.78 \pm 0.14^{*}$ & $0.78 \pm 0.09^{*}$ \\
72 & $0.38 \pm 0.10$ & $0.93 \pm 0.30^{*}$ & $0.81 \pm 0.15^{*}$ \\
96 & $0.28 \pm 0.06$ & $0.71 \pm 0.23^{*}$ & $0.81 \pm 0.12^{*}$ \\
\hline
\end{tabular}

Data presented as mean \pm standard deviation. $R V$, Right ventricular. $* P<.001$ compared with sham group at same measurement point and respective baseline value.
TABLE E3. RVMPI of sham, continuous, and intermittent groups throughout protocol

\begin{tabular}{lccc}
\hline $\begin{array}{c}\text { Measurement } \\
\text { point }(\mathbf{h})\end{array}$ & $\begin{array}{c}\text { Sham } \\
(\mathbf{n}=\mathbf{7})\end{array}$ & $\begin{array}{c}\text { Continuous } \\
(\mathbf{n}=\mathbf{6})\end{array}$ & $\begin{array}{c}\text { Intermittent } \\
(\mathbf{n}=\mathbf{6})\end{array}$ \\
\hline Preoperative & $0.26 \pm 0.15$ & $0.26 \pm 0.09$ & $0.21 \pm 0.05$ \\
0 & $0.24 \pm 0.16$ & $0.26 \pm 0.20$ & $0.19 \pm 0.09$ \\
24 & $0.27 \pm 0.16$ & $0.19 \pm 0.03$ & $0.12 \pm 0.05$ \\
48 & $0.20 \pm 0.12$ & $0.28 \pm 0.09$ & $0.16 \pm 0.09$ \\
72 & $0.16 \pm 0.08$ & $0.38 \pm 0.17^{*}$ & $0.21 \pm 0.13$ \\
96 & $0.22 \pm 0.07$ & $0.41 \pm 0.28^{*}, \dagger$ & $0.10 \pm 0.03$ \\
\hline
\end{tabular}

Data presented as mean \pm standard deviation. RVMPI, Right ventricular myocardial performance index. $* P<.039$ compared with sham group for same measurement point. $\dagger P<.001$ compared with intermittent group for same measurement point.

TABLE E2. RVEF of sham, continuous, and intermittent groups throughout protocol

\begin{tabular}{lcll}
\hline $\begin{array}{c}\text { Measurement } \\
\text { point }(\mathbf{h})\end{array}$ & $\begin{array}{c}\text { Sham } \\
(\mathbf{n}=\mathbf{7})\end{array}$ & $\begin{array}{c}\text { Continuous } \\
(\mathbf{n = 6})\end{array}$ & $\begin{array}{c}\text { Intermittent } \\
(\mathbf{n = 6})\end{array}$ \\
\hline Preoperative & $77.43 \pm 4.89$ & $66.17 \pm 7.28$ & $60.83 \pm 5.34^{*}$ \\
0 & $70.00 \pm 6.27$ & $40.00 \pm 18.38^{*}, \dagger$ & $31.83 \pm 16.35^{*}, \dagger$ \\
24 & $76.14 \pm 5.90$ & $40.50 \pm 15.27^{*}, \dagger$ & $54.83 \pm 13.20^{*}$ \\
48 & $77.29 \pm 6.24$ & $53.20 \pm 21.41^{*}$ & $65.33 \pm 6.19$ \\
72 & $76.00 \pm 6.78$ & $63.00 \pm 16.47$ & $69.00 \pm 8.65$ \\
96 & $74.71 \pm 4.19$ & $63.00 \pm 13.90$ & $72.33 \pm 6.28$ \\
\hline
\end{tabular}

Data presented as \pm standard deviation. $R V E F$, Right ventricular ejection fraction. $* P<.02$ compared with sham group for same measurement point. $\dagger P<.001$ compared with preoperative value. 
TABLE E4. Heart rate of sham, continuous, and intermittent groups at rest and under pharmacologic stress, with respective doses of dobutamine reached by each group

\begin{tabular}{llcccc}
\hline Measurement point & Group & HR at rest $(\mathbf{b p m})$ & Dobutamine dose $(\boldsymbol{\mu g} / \mathbf{k g} / \mathbf{m i n})$ & $\boldsymbol{\Delta} \%$ HR & HR with stress $(\mathbf{b p m})$ \\
\hline Baseline & & & & & \\
& Sham & $134.86 \pm 23.36$ & $31.43 \pm 9.00$ & $60.99 \pm 20.21$ & $206.29 \pm 48.22$ \\
& Continuous & $131.50 \pm 16.88$ & $36.67 \pm 8.16$ & $59.79 \pm 21.05$ & $210.33 \pm 39.70$ \\
& Intermittent & $126.00 \pm 12.70$ & $30.00 \pm 12.60$ & $84.69 \pm 30.50$ & $229.83 \pm 17.50$ \\
$96-\mathrm{h}$ & & & & \\
& Sham & $114.86 \pm 22.93$ & $30.71 \pm 13.67$ & $61.81 \pm 16.11$ & $184.86 \pm 36.10$ \\
& Continuous & $131.83 \pm 14.62$ & $35.00 \pm 8.37$ & $56.16 \pm 29.88$ & $204.67 \pm 36.30$ \\
& Intermittent & $130.00 \pm 16.94$ & $24.17 \pm 13.57$ & $72.20 \pm 7.00$ & $223.33 \pm 24.68$ \\
\hline
\end{tabular}

Baseline, $P=.66$ between groups; $96-\mathrm{h}, P=.10$ between groups. $H R$, Heart rate; $H R$ with stress, heart rate during dobutamine infusion.

TABLE E5. Water content of cardiac masses of sham, continuous, and intermittent groups

\begin{tabular}{lcccc}
\hline $\begin{array}{c}\text { Cardiac mass } \\
(\mathbf{g} / \mathbf{k g})\end{array}$ & Sham $(\mathbf{n}=\mathbf{6})$ & Continuous & Intermittent & $\begin{array}{c}\boldsymbol{P} \\
\text { value }\end{array}$ \\
\hline Right ventricle & $78.84 \pm 2.41$ & $82.00 \pm 1.11^{*}$ & $81.85 \pm 1.41^{*}$ & .0102 \\
Septum & $77.11 \pm 2.08$ & $80.04 \pm 0.27 *$ & $79.53 \pm 0.75^{*}$ & .0028 \\
Left ventricle & $78.20 \pm 1.89$ & $79.23 \pm 0.40$ & $79.08 \pm 0.69$ & .2887 \\
\hline \multicolumn{5}{l}{ Data presented as mean \pm standard deviation. ${ }^{*} P<.01$ compared with sham group. }
\end{tabular}

